\title{
Bayesian Networks for Incorporation of Contextual Information in Target Recognition Systems
}

\author{
Keith Copsey and Andrew Webb
}

\author{
QinetiQ \\ St Andrews Road, Malvern, Worcs, WR14 3PS, UK \\ K.Copsey@signal.QinetiQ.com \\ webb@signal.QinetiQ.com
}

\begin{abstract}
In this paper we examine probabilistically the incorporation of contextual information into an automatic target recognition system. In particular, we attempt to recognise multiple military targets, given measurements on the targets, knowledge of the likely groups of targets and measurements on the terrain in which the targets lie. This allows us to take into account such factors as clustering of targets, preference to hiding next to cover at the extremities of fields and ability to traverse different types of terrain. Bayesian networks are used to formulate the uncertain causal relationships underlying such a scheme. Results for a simulated example, when compared to the use of independent Bayesian classifiers, show improved performance in recognising both groups of targets and individual targets.
\end{abstract}

\section{Introduction}

In this paper we examine probabilistically the incorporation of contextual information and domain specific knowledge into an automatic target recognition (ATR) system for military vehicles.

In a realistic ATR scenario, after an initial detection phase, there will be a set of multiple locations which have been identified as possibly occupied by targets/vehicles. Appropriate measurements (e.g. radar, sonar, infra-red, etc) will then be taken at each of these locations, so that classifications can be made. Some of the measurements might be from real targets, while others will be false alarms from clutter objects, or just background noise. Most standard ATR techniques[7] will consider each potential target independently.

In this work, we look at how, in a military scenario, the posterior probabilities of class membership at each location, can be combined with additional domain specific knowledge. This reflects the fact that a human assigning classes to measurements would take into account contextual information as well as the data measurements themselves. The use of this sort of additional contextual information by an operator might be stronger than just having a closer look at the data measurements in certain locations; it may tip the balance towards (or 
away from) certain classes. Thus, two nearly identical measurements may actually be assigned to different classes, depending on their respective contextual information.

The type of contextual information that can be incorporated could include the proximity of other vehicles, recognising that military targets will often travel in groups. A human operator might also pay more attention to the extremities of fields close to hedges and woodland edges, reflecting the fact that military commanders would consider their vehicles exposed in the centre of a field and might choose to get them as close to cover as possible. Further domain specific knowledge that could be brought to the problem includes the type of terrain that surrounds the target and knowledge about the likely deployment of military vehicles, such as formations.

Simply altering our set of possible classes, based on the contextual information, is not appropriate, since there are almost always going to be uncertainties. For example, our estimates of the surrounding terrain might be in error. The most appropriate formalism for handling the possibly conflicting pieces of information in a consistent manner is probabilistic. Thus, conventional expert systems [5] are not appropriate. However, a Bayesian network[3, 4] based on the causal relationships leading to a deployment of targets within a region, can be used in a probabilistic way to integrate domain specific knowledge with the actual data measurements.

\subsection{Bayesian Networks}

Bayesian networks (also referred to as belief networks, Bayesian graphical models and probabilistic expert systems) can be used to model situations in which causality plays a role, but where there is a need to describe things probabilistically, since our understanding of the processes occurring is incomplete or, indeed, the processes themselves have a random element.

A Bayesian network can be illustrated graphically as a series of nodes, which may assume particular states, connected by directional arrows. Figure 1 shows such a network. The states of a node can be discrete or continuous. Every node has an associated conditional probability table or density, specifying the probability or probability density of the node being in a certain state given the states of the nodes with arrows pointing towards it. Nodes with no arrows pointing towards them are assigned prior distributions over their states. Given observations on some of the nodes, beliefs are propagated up through the network to obtain posterior probabilities for the unobserved nodes.

\subsection{Problem Definition}

We focus on the situation where (after an initial detection phase) there is a set of objects at estimated locations, with each object being a potential target/vehicle. Each of these objects needs to be assigned to a class, i.e. either a specific class of target or just clutter. A single multi-dimensional measurement of each object (i.e. at each location) is available, as well as estimates of the terrain of the 
region surrounding the objects. This terrain estimate consists of the division of the overall region into sub-regions, each with an associated local terrain (e.g. field, marsh, etc). These sub-regions are separated by boundaries, which are of unspecified type. The boundaries are allowed to split sub-regions of the same local terrain, so fields split by hedges or walls are treated as separate sub-regions.

In our work we consider a subset of the contextual or domain specific information that can be incorporated into such an ATR problem. In particular, we focus on the proximity of other vehicles, the distances to boundaries, the immediate type of terrain and known groupings of targets.

\subsection{Related Work}

A related approach to the work documented here is given by Blacknell[1], who looked at the incorporation of contextual information in SAR target detection, by altering the prior probabilities of targets, depending on terrain, the distances to boundaries and the proximity of other potential targets. The use of Bayesian networks to exploit contextual information for vehicle detection in infrared linescan imagery has been reported by Ducksbury et al[2]. Musman and Lehner[6] use Bayesian networks to schedule a shipboard self-defence mechanism.

\section{Incorporation of Domain Specific Knowledge}

Our proposed Bayesian network is illustrated in Fig. 1. The nodes denote states of affairs and the arcs can be interpreted as causal connections.

The "groups" node represents the collections/groups that targets are likely to be deployed from, while the "terrain" node represents the terrain over the region

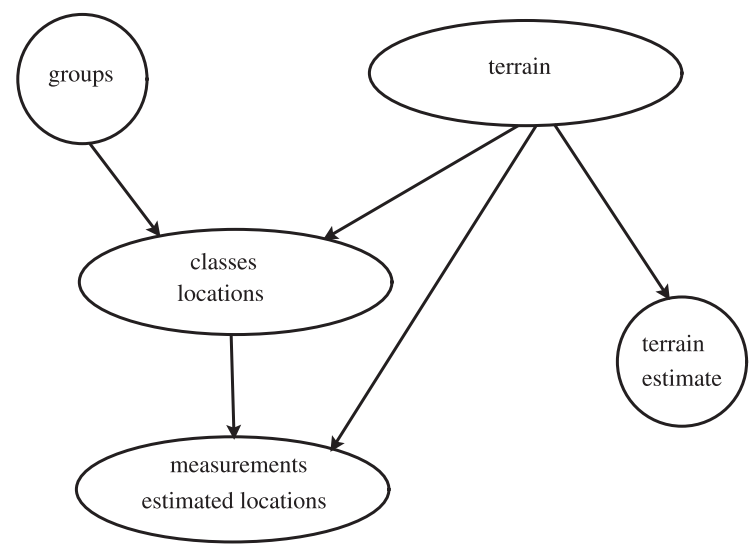

Fig. 1. Bayesian network formulation for the incorporation of contextual information in an ATR system 
of interest. The "terrain estimate" node is made up of our observations of the terrain in the region of interest, based, for example, on Synthetic Aperture Radar (SAR) image analysis. The node labelled "classes" and "locations" represents the classes and locations of the objects, whereas the node labelled "measurements" and "estimated locations" contains our measurements of the objects and our estimates of the locations.

In our scenario, after an initial detection phase, we have a set of $n_{l}$ potential target locations, $\hat{l}=\left\{\hat{l}_{i} ; 1 \leq i \leq n_{l}\right\}$, with corresponding data measurements $x=\left\{x_{i} ; 1 \leq i \leq n_{l}\right\}$.

There are $J \geq 1$ target types and these are supplemented with a noise/clutter class, giving, in total, $J+1$ classes. The measurements at the potential target locations can, therefore, be referred to as coming from a collection of $n_{l}$ objects, each of which belongs to one of the $J+1$ classes.

\subsection{Groups of Targets}

Deployed targets are taken to come from specific groups of targets/vehicles, which we denote by the discrete random variable, $G$. The cover of $G$ is the set of possible groups, which is assigned using expert knowledge. If the number of targets within a group is less than the number of potential target locations, $n_{l}$, the group is augmented with clutter measurements. The prior probabilities for the states of $G$ would, ideally, be assigned using intelligence information.

\subsection{Terrain}

The random variable representing the terrain, denoted $T$, is a broad variable covering many aspects and is made up of both continuous and discrete elements. In our case, this includes the positions of the boundaries of the sub-regions of the area of interest and the local terrain types within each sub-region. We suppose that the local terrain types must belong to a discrete set of $n_{\tau}$ terrain types, such as "field", "urban", "marsh" etc.

Attempting to assign decent prior distributions for the constituents of $T$ is a hard task, since the number and locations of the sub-regions are random variables. Assigning flat priors is possible, but this makes later inference awkward, since the states have to be integrated over. However, progress can be made if our conditional distribution for the observation of the terrain given the actual terrain is restrictive. This is covered in Section 2.5.

\subsection{Classes and Locations}

The random variable $C$ denotes the classes at each of the $n_{l}$ potential target locations. A state of $C$ consists of a $n_{l}$-dimensional vector, $c=\left(c_{1}, \ldots, c_{n_{l}}\right)$, with elements the classes for each of the $n_{l}$ objects. The class allocations variable is coupled with the locations variable, $L$, which contains the actual locations of the objects. A state of $L$ consists of a $n_{l}$-dimensional vector, $l=\left(l_{1}, \ldots, l_{n_{l}}\right)$, with 
elements the locations for each of the $n_{l}$ objects. A pair $(C, L)=(c, l)$ is referred to as an allocation of targets.

The conditional distribution for this node of the Bayesian network is, for ease of notation, denoted by $p(c, l \mid g, t)$. Its specification (Section 2.6) allows incorporation of contextual information.

\subsection{Measurements and Locations}

The measurements, $x$ and estimated locations, $\hat{l}$, depend on the actual classes and locations, $(C, L)=(c, l)$ and the terrain $T=t$, via the conditional distributions, $p(x, \hat{l} \mid c, l, t)$.

We take the data measurements to be conditionally independent and assume:

$$
p(x, \hat{l} \mid c, l, t)=\prod_{i=1}^{n_{l}} p\left(x_{i} \mid c_{i}, l_{i}, t\right) p\left(\hat{l}_{i} \mid l_{i}, t\right) .
$$

The distribution $p\left(x_{i} \mid c_{i}, l_{i}, t\right)$ is the measurement distribution for the class, $c_{i}$, in the terrain, $t$, at location, $l_{i}$. In practice we frequently take this distribution to be independent of the terrain and given by $p\left(x_{i} \mid c_{i}\right)$, although this is not necessary in our Bayesian network approach. Specification of these distributions is, of course, very difficult and the subject of much research interest[7].

The distribution $p\left(\hat{l}_{i} \mid l_{i}, t\right)$ is generally taken to be a $\delta$-function, $p\left(\hat{l}_{i} \mid l_{i}, t\right)=$ $\delta\left(\hat{l}_{i}-l_{i}\right)$, so that the measured locations are the same as the actual locations. This simplification is for computational reasons in specifying the conditional probability distributions, $p(c, l \mid g, T)$ and in propagating evidence in the network.

A standard Bayesian classifier comprises only the distributions $p\left(x_{i} \mid c_{i}\right)$, along with some very simple prior probabilities for the classes, $p\left(c_{i}\right)$. Classifications are made to the maximum a posteriori (MAP) class, as determined by the posterior class probabilities from Bayes' rule, $p\left(c_{i} \mid x_{i}\right) \propto p\left(x_{i} \mid c_{i}\right) p\left(c_{i}\right)$.

\subsection{Terrain Estimate}

The random variable $\hat{T}$ representing our estimates of the terrain, depends on the actual terrain via the conditional distribution $p(\hat{T} \mid T)$. Similarly to Section 2.2, the terrain estimate consists of the positions and boundaries of sub-regions, along with their respective local terrain types.

A full treatment of the possible states (and associated conditional distribution) is not feasible, because of the requirement to specify the distribution $p(c, l \mid g, t)$ for each allowable $t$. Thus, we are forced to assume that the number of sub-regions is correctly observed, as are the boundaries of these subregions. However, the observations/estimates of the local terrain types within these boundaries can be erroneous. Thus, the conditional distribution, $p(\hat{T} \mid T)$, is defined using a $n_{\tau} \times n_{\tau}$ matrix of the conditional probabilities of the terrain type estimates given the actual terrain types. This matrix would be determined by consultation with experts, who could take into account the techniques used to estimate local types of terrain. 


\subsection{The Conditional Distributions of Target Allocations}

If suitable training data were available, the conditional distribution, $p(c, l \mid g, t)$, could be learnt from the data[3, 4]. However, the availability of such data is often quite limited, so we rely on expert opinion and representative values to determine the distribution.

Our conditional distribution is expressed as a product of weights:

$$
p(c, l \mid g, t) \propto w_{\text {bndry }}(c, l, t) \times w_{\text {clust }}(c, l, t) \times w_{\text {terr }}(c, l, t) \times w_{\text {grp }}(c, l, g, t),
$$

where $w_{\text {bndry }}(c, l, t)$ is a factor related to the distances of vehicles from boundaries, $w_{\text {clust }}(c, l, t)$ is a factor related to the clustering of targets, $w_{\text {terr }}(c, l, t)$ is a factor related to the local types of terrain at the object locations and $w_{\operatorname{grp}}(c, l, g, t)$ is a factor relating the allocation defined by $(c, l)$ to the group of targets $g$. Due to a lack of space we do not go into the details of these weighting factors.

\section{Using the Bayesian Network}

Having specified our Bayesian network we need to be able to calculate the required posterior distributions of the nodes of the network, based on our measurements, $(x, \hat{l}, \hat{t})$. This is referred to as updating the beliefs in a Bayesian network. There are a number of ways of making inference on Bayesian networks and these are well documented in $[3,4]$. Whatever the method, the posterior node distributions of interest are $p(g \mid x, \hat{l}, \hat{t}), p(c, l \mid x, \hat{l}, \hat{t})$ and $p(c, l \mid g, x, \hat{l}, \hat{t})$. These correspond to the marginal posterior probabilities of the groups; the marginal posterior probabilities for the allocations; and the marginal posterior probabilities for the allocations conditional on the group.

In this paper we use a "long-hand" approach for belief updating, which explicitly carries out the summations required for each marginal or conditional posterior distribution of interest. This has the advantage of being quick to program and is exact. However, as the number of states in the network increases, the direct summation approach to updating beliefs becomes computationally complex (the computational cost increases exponentially with the number of objects to be examined).

\subsection{Using the Probabilities}

The posterior probabilities contain our updated beliefs about the objects and should be incorporated into a model that reflects the whole decision making process. By considering the expected posterior loss of decisions, account can be made of the different costs involved in making erroneous decisions. However, for the purposes of this paper, single classifications are proposed, based on the MAP probabilities of interest.

The most likely group is taken to be the group, $\hat{g}$, that maximises the probability $p(g \mid x, \hat{l}, \hat{t})$. The most likely allocation of classes is the allocation maximising the probability $p(c, l \mid x, \hat{l}, \hat{t})$. Also of interest is the allocation maximising $p(c, l \mid \hat{g}, x, \hat{l}, \hat{t})$. 


\subsection{Standard Bayesian Classifier}

For the standard Bayesian classifier (described in Section 2.4), determination of the most likely group is awkward. To assess the performance of the Bayesian network in determining groups we have therefore invented a simple group assignment scheme for the standard Bayesian classifier. In particular, we assign a set of objects to belong to a specific group, if all the targets in the group are present within the set of classes from the MAP class probabilities for the objects.

\section{Simulated Example}

\subsection{Scenario}

The performance of the Bayesian network approach (compared to a standard Bayesian classifier) is illustrated with a simulated example.

We consider a problem with three target classes, namely main battle tanks (MBTs), armoured personnel carriers (APCs) and air defence units (ADUs), which on addition of the clutter class gives a four class problem. Target deployments are taken to come from three possible groupings. The 1st group, $g_{1}$, consists of $3 \mathrm{MBTs}$ and an APC. The 2nd group, $g_{2}$, consists of 4 APCs and the 3rd group, $g_{3}$, consists of 2 ADUs and an APC. We consider the situation where intelligence information indicates that group $g_{1}$, is more likely than group $g_{2}$, which is, in turn, more likely than group $g_{3}$.

For demonstration purposes, the measurement distributions are taken to be given by 2-dimensional Gaussian distributions, which have been chosen to overlap. In our experiments, these measurement distributions are assumed known. This affects both the Bayesian network and the standard Bayesian classifier. It is, of course, unrealistic in an actual ATR scenario, but allows us to focus on the effects of the contextual information.

A terrain has been simulated, dividing the area of interest into five subregions (each of which has a local terrain type). Scenarios of target deployments following our expected target behaviour (i.e. travelling together and close to cover) for each of the three groups have been simulated, along with some additional clutter objects. These scenarios are illustrated in Fig. 2. In each case, 5 objects have been picked up in the (unspecified) initial detection phase. For scenario 1 (a deployment for $g_{1}$ ), from top to bottom the objects and their respective classes are O-5 (Clutter), O-4 (APC), O-1, O-3 and 0-2 (all MBTs), respectively. For scenario 2 (a deployment for $g_{2}$ ), from top to bottom the objects are O-5 (Clutter), O-1, O-4, O-2 and 0-3 (all APCs). Finally, for scenario 3 (a deployment for $g_{3}$ ), from top to bottom the objects are 0-2 (ADU), O-3 (APC), O-4 (Clutter), O-1 (ADU) and O-5 (Clutter).

For each of the three scenarios, 500 sets of measurements for the objects have been simulated. Performance within each scenario is estimated from the results for that scenario's sets of measurements. 

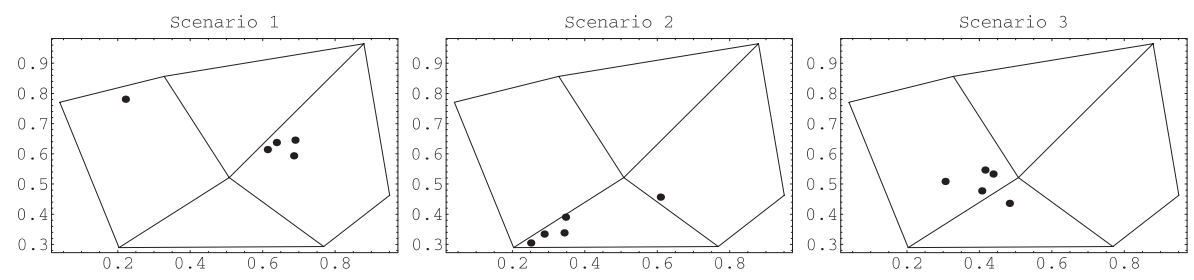

Fig. 2. Scenario for each of the three groups, with objects marked by dots

\subsection{Experimental Results}

The percentages of correctly identified groups for the Bayesian network are $85.0 \%, 90.2 \%$ and $63.0 \%$ for the 1st, 2 nd and $3 \mathrm{rd}$ scenarios respectively. This outperforms significantly the corresponding standard Bayesian classifier results of $16.0 \%, 48.2 \%$ and $56.6 \%$.

In Fig. 3 we show the classification rates for each of the five objects, in each of the three scenarios (with the underlying classes of the objects detailed in Section 4.1). For the first two scenarios, the Bayesian network approaches can be seen to out-perform the standard approach significantly. For the third scenario, the standard approach marginally out-performs the Bayesian networks for the first two objects (ADUs) and the clutter objects, but the Bayesian network approaches have better performance on the APC. There is little to choose between the two Bayesian network approaches.

The poorer performance of the network on the 3rd scenario, in terms of improvement over the the standard classifier, is an artefact of the lower prior probability assigned to the 3rd group (which comes from the simulated intelligence information that the 3rd group is less likely than the other two groups).
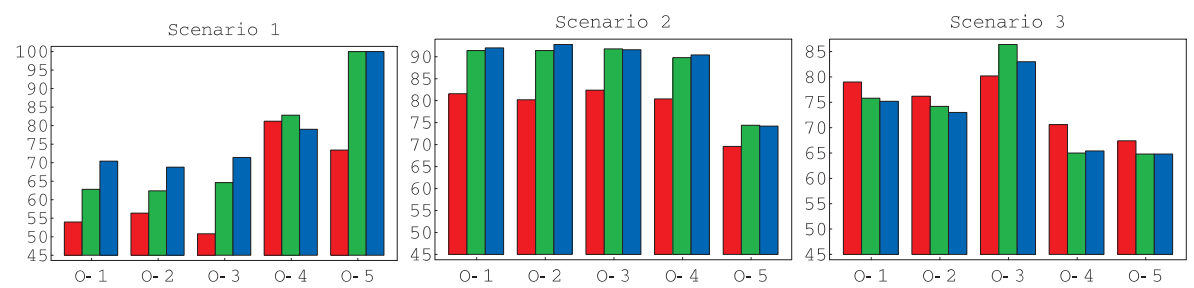

Fig. 3. The classification rates for the two techniques based on the Bayesian network, compared to the standard Bayesian classifier. For each object and scenario, from left to right, the bars correspond to the standard classifier, the Bayesian network with MAP $p(c, l \mid x, \hat{l}, \hat{t})$, and the Bayesian network with MAP $p(c, l \mid \hat{g}, x, \hat{l}, \hat{t})$ 


\section{Summary and Discussion}

In this work we have shown how Bayesian networks can be used to incorporate domain specific knowledge and contextual information into an ATR system, used for multiple target recognition of military vehicles. Given measurements on the terrain in which the targets lie, we have taken into account such factors as clustering of targets, preference to hiding next to cover at the extremities of fields and the varying abilities of vehicles to traverse different types of terrain. These have been combined in a consistent probabilistic manner, with the information contained in measurements of the targets and with prior knowledge on the groupings of targets. A potential area for further research is the incorporation of other contextual factors into the system, the major difficulty lying in the translation of the factors into appropriate conditional probability distributions.

In a simulated scenario, the Bayesian network has been shown to outperform classification using a standard Bayesian classifier (which uses only the target measurements), both in terms of recognising groups of targets and the performance at specific locations. Currently, the technique has been tested only on simulated data. Future research will need to assess the approach on real data.

\section{Acknowledgments}

This research was sponsored by the UK MOD Corporate Research Programme. CC Copyright QinetiQ 2002.

\section{References}

[1] D. Blacknell. Contextual information in SAR target detection. IEE Proceedings - Radar, Sonar and Navigation, 148(1):41-47, February 2001. 711

[2] P. G. Ducksbury, D. M. Booth, and C. J. Radford. Vehicle detection in infrared linescan imagery using belief networks. Proceedings of 5th International Conference on Image processing and its applications, Edinburgh, UK, July 1995. 711

[3] F. V. Jensen. Introduction to Bayesian Networks. Springer-Verlag, 1997. 710, 714

[4] M. I. Jordan. Learning in Graphical Models. The MIT Press, February 1999. 710, 714

[5] G. Luger and W. Stubblefield. Artificial Intelligence: Structures and Strategies for Complex Problem Solving. Addison Wesley, 2nd edition, 1993. 710

[6] S. Musman and P. Lehner. Real-time scheduling under uncertainty for ship self defence. Submitted to IEEE Expert Special Issue on Real-time Intelligent Systems, 1998. 711

[7] A. R. Webb. Statistical Pattern Recognition. John Wiley \& Sons, Chichester, 2nd edition, August 2002. 709, 713 